\title{
Estudio de perfiles de difracción de rayos $X$ de una aleación Ti-13Ta-3Sn obtenida por aleado mecánico
}

\section{Study of X-ray diffraction profiles of a Ti-13Ta-3Sn alloy obtained by mechanical alloying}

Edgar Ivan Pio Lopez ${ }^{1}$, Pablo Ignacio Martin Saint-Laurence ${ }^{1}$, Claudio Eduardo Aguilar Ramirez ${ }^{1}$, Luis Bejar Gomez ${ }^{2}$, Ariosto Medina Flores ${ }^{3}$, Fernando De Las Cuevas Jimenez ${ }^{4}$, Ismeli Alfonso López ${ }^{5}$

\footnotetext{
${ }^{1}$ Departamento de Ingeniería Metalúrgica y Materiales, Universidad Técnica Federico Santa María, Av. España 1680, Valparaíso, Chile.

${ }^{2}$ Facultad de Ingeniería Mecánica, Universidad Michoacana de San Nicolás de Hidalgo, Edificio W. Ciudad Universitaria, Morelia, Michoacán, México.

${ }^{3}$ Instituto de Investigaciones en Metalurgia y Materiales, Universidad Michoacana de San Nicolás de Hidalgo, Ciudad Universitaria, Morelia, Michoacán, México.

${ }^{4}$ Departamento de Ciencias, Universidad Pública de Navarra, Pamplona, Navarra, España.

${ }^{5}$ Instituto de Investigaciones en Materiales, Unidad Morelia, Universidad Nacional Autónoma de México, Campus Morelia UNAM. Antigua Carretera a Pátzcuaro No. 8701, Col. Ex -Hacienda de San José de la Huerta. CP: 58190, Morelia, Guayangareo, Michoacán, México.

e-mail: edgar.pio@sansano.cl,pablo.martin12@sansano.usm.cl, claudio.aguilar@usm.cl, lbejargomez@yahoo.com.mx, arimedflo@yahoo.com.mx, fernando.delascuevas@unavarra.es, ialfonso@iim.unam.mx
}

\section{RESUMEN}

Las aleaciones Ti- $\beta$ se han vuelto altamente demandadas en la industria, por sus buenas características físicas y químicas. En el presente trabajo, se sintetizó una aleación Ti-13Ta-3Sn (\%at) por aleado mecánico con tiempos entre 2 y 100h. Las aleaciones se caracterizaron por difracción de rayos X (DRX) y los patrones se analizaron por el método Rietveld con el software MAUD. Se caracterizaron los cambios microestructurales y la evolución de las fases Ti- $\alpha$ y Ti- $\beta$. Se identificó la presencia una nueva fase metaestable fcc y la síntesis de una aleación con un 79,80\% en peso de fase Ti- $\beta$, ambas con tamaño de cristalita nanométrico.

Palabras clave: Aleado mecánico, aleación Ti- $\beta$, difracción de rayos X.

\section{ABSTRACT}

The Ti- $\beta$ alloys have become highly demanded in industry, due to their good physical and chemical characteristics. In the present work, a Ti-13Ta-3Sn (\%at) alloy was synthesized using mechanical alloying (MA) between the times of 2 and 100h. The alloys were characterized by X-ray diffraction (XRD) and analyzed by the Rietveld method with the MAUD software. The microstructural changes and the evolution of the Ti- $\alpha$ and Ti- $\beta$ phases were characterized. Identifying the presence of a new metastable fcc phase and the synthesis of an alloy with $79.80 \%$ wt of Ti- $\beta$ phase, both alloys with nanometric crystallite size.

Keywords: Mechanical alloying, Ti- $\beta$ alloy, X-ray diffraction. 


\section{INTRODUCCIÓN}

De acuerdo con los datos del Banco Mundial, la expectativa de vida aumentó de 52 a 71 años en el período de 1960 a 2016 [1]. Uno de los principales problemas asociados a este incremento de edad es la osteoporosis, fenómeno que se presenta a edades superiores a 50 años de acuerdo con cifras de la Fundación Internacional de Osteoporosis (IOF) [2-5]. La osteoporosis provoca más de 8,9 millones de fracturas al año, resultando en una fractura osteoporótica cada 3 segundos [6].

En la actualidad los implantes base titanio están constituido por las aleaciones Ti-6Al-4V y Ti-Ni. Sin embargo estudios recientes, han demostrado que la presencia de vanadio (V) en los tejidos humanos, puede alterar la respuesta de la actividad enzimática inflamatoria de las células [2, 3] y la presencia del aluminio (Al) a largo plazo, aumenta la posibilidad del desarrollo de Alzheimer [4, 5]. También la presencia de níquel (Ni) causa una reacción alérgica en el cuerpo humano [6, 7]. Lo anterior, significa que se deben desarrollar nuevas aleaciones base titanio sin la presencia de los elementos antes mencionados. Es importante que los biomateriales metálicos base Ti cumplan, con las características mecánicas requeridas por el hueso humano, es decir, valores de módulo elástico entre 0,4 a $30 \mathrm{GPa}$ [8,9] y esfuerzo de fluencia sobre $115 \mathrm{MPa}$ [15].

Las aleaciones base Ti se pueden clasificar en 3 grupos: alfa $(\alpha)$ con estructura cristalina hexagonal compacta (hcp), beta ( $\beta$ ) con estructura cubica centrada en el cuerpo (bcc) y alfa-beta $(\alpha-\beta)$ con ambas estructuras cristalinas. Para estabilizar la fase $\alpha$ se emplean Al, O, N, C, para estabilizar la fase $\beta$ se usa V, Nb, Ta, Mo (isomorfo), Fe, W, Cr, Ni, Si, Co, Mn, H (eutectoide), y los elementos neutros son (Zr y Sn [11, 12]). Las aleaciones de tipo $\beta$, son preferidas para aplicaciones biomédicas porque presentan bajos valores de modulo elástico $[8,13,14]$ y buena resistencia a la corrosión $[15,16]$.

Considerando la clasificación anterior, es posible seleccionar los elementos estabilizadores y neutros, que cumplan con características de biocompatibilidad, de acuerdo con los trabajos realizados por Steimemann y Kawahara [3,17] (Figura 1). Así, los candidatos pueden incluir el Nb, Zr, Mo, Ta y Sn, siendo los últimos dos, los mejores en biocompatibilidad [18-20].
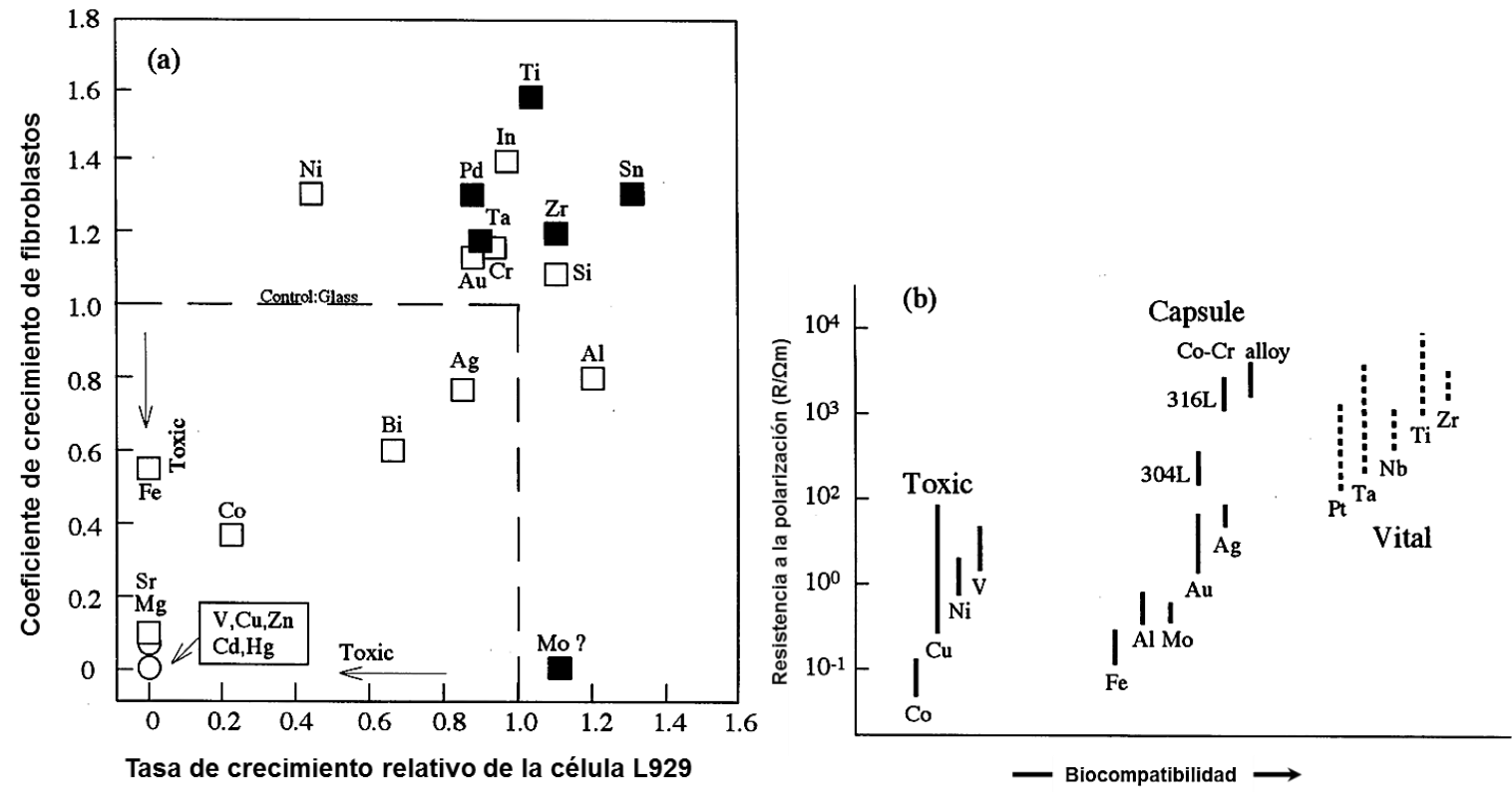

Figura 1: (a) Citotoxicidad de metales puros y (b) Relación entre la resistencia a la polarización y la biocompatibilidad de metales puros, aleación Co-Cr y aceros inoxidables $[3,17]$. 
Uno de los procesos de producción de aleaciones y compuestos es el aleado mecánico (AM), este método fomenta la difusión de átomos a través de constantes deformaciones, uniones y fracturas de los polvos metálicos, cerámicos o polímeros [26]. Por medio de AM se han reportado soluciones sólidas de varios sistemas, tales como, lo reportado por Schaffer et al.[27] quien estudio la evolución de la reacción de CuO-Fe en un molino de alta energía, Benjamin et al.[28] describió detalladamente la evolución la aleación Cr-Fe en molienda de alta energía y Lu et al.[29] quien propuso después de diversos experimentos los principales factores en la formación de solución sólida. También es posible obtener compuestos intermetálicas [26-28], alecciones metaestables [29-31] y la formación de materiales amorfos [32-34]. Autores como Rojas et al. [39], analizo los patrones de difracción de rayos X para determinar cambios en el parámetro de red, microdeformaciones y tamaño de cristalita en aleaciones de $\mathrm{Cu}$ obtenidas por $\mathrm{AM}$, Ma y Atzmon [35] con el mismo método observaron la evolución de las fases las aleaciones Zr-Al y Fe-Cu, Oehringz [34] la estabilidad de la aleación de Ti-Si y Ti-Al obtenidas por AM. Pelegrini [40] identificó la transformación de fases en la aleación Fi-Si-P mediante el análisis de los patrones de difracción de los rayos X. Al comparar la evolución de los patrones de difracción de rayos $\mathrm{X}$ en función del tiempo de molienda, identificó la evolución microestructural y el tiempo en el que se genera la transformación de fase.

De acuerdo con lo anterior es importante, identificar el tiempo en el que se inicia la transformación de la fase desde Ti- $\alpha$ a Ti- $\beta$, así como el tiempo óptimo en el cual se presenta la máxima cantidad de fase Ti- $\beta$. Por ello el objetivo del presente trabajo se enfoca en el estudio de la evolución de fases por medio de análisis de perfiles de difracción de rayos $\mathrm{X}$ durante la molienda de la aleación Ti-13Ta-3Sn. Se aplicó el método Rietveld para la cuantificación de fases y determinar parámetros microestructurales.

\section{MATERIALES Y MÉTODOS}

Se usaron polvos metálicos comercialmente puros (cp) de Ti grado IV $(<149 \mu \mathrm{m}$, de la empresa Noah Technologies), polvos cp de Ta (con una pureza del 99,9\% y malla -325, de la compañía Sigma-Aldrich) y polvos cp de Sn (con pureza del 99,8\% y malla 100, de la compañía Sigma-Aldrich). Se usó una mezcla de Ti-13Ta3Sn (\% atómico). Los medios de molienda utilizados (contenedores y bolas), fueron de $\mathrm{ZrO}_{2}$ estabilizada con itrio (YSZ), con una capacidad de $250 \mathrm{ml}$. Se usaron dos diferentes diámetros de bolas (11 y $5 \mathrm{~mm}$ ). La molienda se hizo en un molino planetario Retsch PM400 a una velocidad $250 \mathrm{rpm}$.

Para evitar la oxidación de la aleación, los contenedores se cargaron en una cámara de guantes con atmosfera de gas Argón (pureza del 99,999\% con <2 ppm de $\mathrm{O}_{2}$ ). Para minimizar la contaminación se realizó un recubrimiento de titanio con una molienda de $2 \mathrm{~h}$ a $250 \mathrm{rpm}$. Los contenedores fueron cargados con una mezcla de Ti-13Ta-3Sn y 2\% en peso de ácido esteárico como agente de control del proceso (ACP). Se usó una relación bola-polvo de 10:1 (100g de bolas de $11 \mathrm{~mm}$ y $100 \mathrm{~g}$ de bolas de $5 \mathrm{~mm}$ ). Para poder evitar el aumento de temperaturas, se utilizó un sistema de aire forzado y ciclos de encendido y apagado de 30 y 30 minutos, respectivamente. Se retiraron muestras de polvos $(0,125 \mathrm{~g})$ en los intervalos de 2, 5, 10, 15, 20, 30, 50 y 100 horas.

Los patrones de difracción de rayos $\mathrm{X}$ fueron obtenidos utilizando un difractómetro de polvo multipropósito STOE STADI MP, equipado con un detector DECTRIS MYTHEN $1 \mathrm{~K}$ con radiación de CuK $\mathrm{K}_{\alpha 1}$ $(\lambda=0.15406 \mathrm{~nm})$. Las muestras se midieron en modo de transmisión usando un paso de $0,12^{\circ}$ y un tiempo por paso de 10 segundos, en un rango angular $2 \theta$ de $20^{\circ}$ a $120^{\circ}$. Se usó el software Materials Analysis Using Diffraction (MAUD) [37, 38] para hacer refinamiento Rietveld y obtener parametros microestructurales como parámetro de red, tamaño medio de la critalita $(\langle\mathrm{D}\rangle)$, cuadrado de la raíz media de la microdeformación $\left(\left\langle\varepsilon^{2}\right\rangle^{1 / 2}\right)$ y la cuantificación de las fases. Se utilizó un standard de $\mathrm{LaB}_{6}(\mathrm{a}=4,1565915(1) \AA)$ para la corrección del error instrumental [43]. 


\section{RESULTADOS}

Los patrones de difracción de rayos X de la aleación Ti-13Ta-3Sn (\% at.) molida entre 2 a 100 horas son mostrados en la Figura 2. Para el tiempo de 2 horas de molienda se observaron los picos de difracción de los polvos elementales (Ti (hexagonal compacta (hcp), grupo espacial $\mathrm{P} 63 / \mathrm{mmc}$ ), Ta (cubica centrada en el cuerpo (bcc), Im $\overline{3} \mathrm{~m}$ ) y Sn (tetragonal centrada en el cuerpo (bct), I4/amd:1)). A su vez se identificaron otras dos fases, una de TiTaSn hexagonal compacta (hcp) (Figura 2 (O)) y otra de TiTaSn centrada en el cuerpo (bcc) (Figura $2(\boldsymbol{\square})$ ), indicando la formación de solución solida de las aleaciones Ti- $\alpha$ y Ti- $\beta$ respectivamente.

Al incrementar el tiempo de molienda a 5h, se observaron los siguientes cambios, i) la ausencia de los picos de difracción del Sn, confirmando la formación estas soluciones sólidas, ii) desplazamientos de los picos de las fases Ti- $\alpha$ y Ti- $\beta$ debido al cambio de parámetro de red y iii) la aparición de nuevos picos, los cuales se identificaron como una estructura centrada en las caras (fcc) y grupo espacial Fm $\overline{3} \mathrm{~m}$.

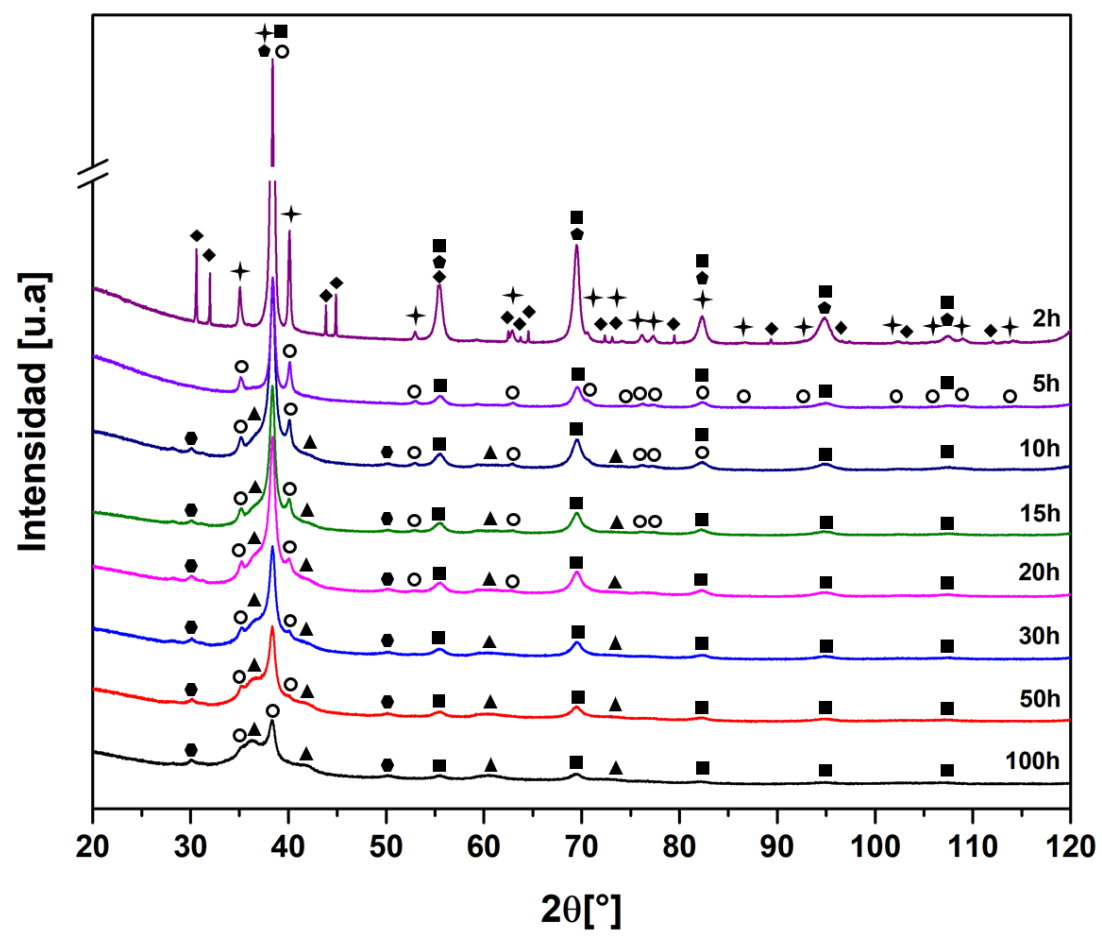

Figura 2: Patrones de difracción de rayos X, de las aleaciones de Ti13Ta3Sn molidas por 2 a 100 horas. ( $\bullet) b c t$-Sn (GE,

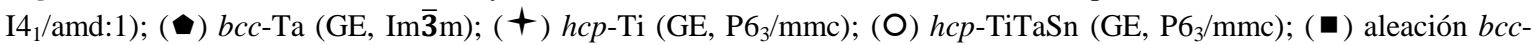

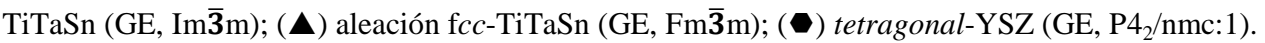

A medida que aumenta el tiempo de molienda se observan tres fases, la primera hcp-TiTaSn, (fase $\alpha$ ), la segunda bcc-TiTaSn, (fase $\beta$ ), y la tercera fase fcc-TiTaSn (o fase $\gamma$ ). La presencia de estas tres fases se mantuvo en todo el proceso de AM, con una variación en la cantidad de cada una de las fases en función del tiempo de molienda, manteniendo un error promedio del 0,15\% en el análisis, Figura 3. Para la muestra de 10h, al analizar los resultados de DRX con el método Rietveld, se observó el incremento de la fase TiTaSn- $\beta$ y TiTaSn- $\gamma$, mientras que la fase TiTaSn- $\alpha$ disminuyó, como se refleja en la Figura 3. Esto muestra que hay una transformación del tipo $\alpha \rightarrow \beta$. Se identificó también la presencia de contaminación de zirconia estabilizada con itria (YSZ) con una estructura tetragonal simple (st) y grupo espacial P4 $/ \mathrm{nmc} 1$, (Figura 2), con una presencia del 1,60\% en peso. Entre 15 y 100 h, se presentó disminución de la fase TiTaSn- $\alpha$ (la Figura 3), lo cual está asociado a una de las siguientes transformaciones; i) $\alpha \rightarrow \beta$ y ii) $\alpha \rightarrow \gamma$. A 30h se observa que la máxima cantidad de fase TiTaSn- $\beta$, fue de un $79,80 \%$ en peso, predominando la transformación de fases $\alpha \rightarrow \beta$. Posteriormente, a las 50h se identificó un incremento de la fase TiTaSn- $\alpha$ y TiTaSn- $\gamma$, lo que muestra que ocurren las siguientes transformaciones reportadas en literatura, $\beta \rightarrow \alpha$ [44] y $\beta \rightarrow \gamma$ [45]. Finalmente, a 100h, se observó un incremento en TiTaSn- $\beta$ y TiTaSn- $\gamma$, indicando que ocurren las transformaciones $\alpha \rightarrow \beta$ y $\alpha \rightarrow \gamma$, inducida por las altas deformaciones causadas por el aleado mecánico [46, 47]. 


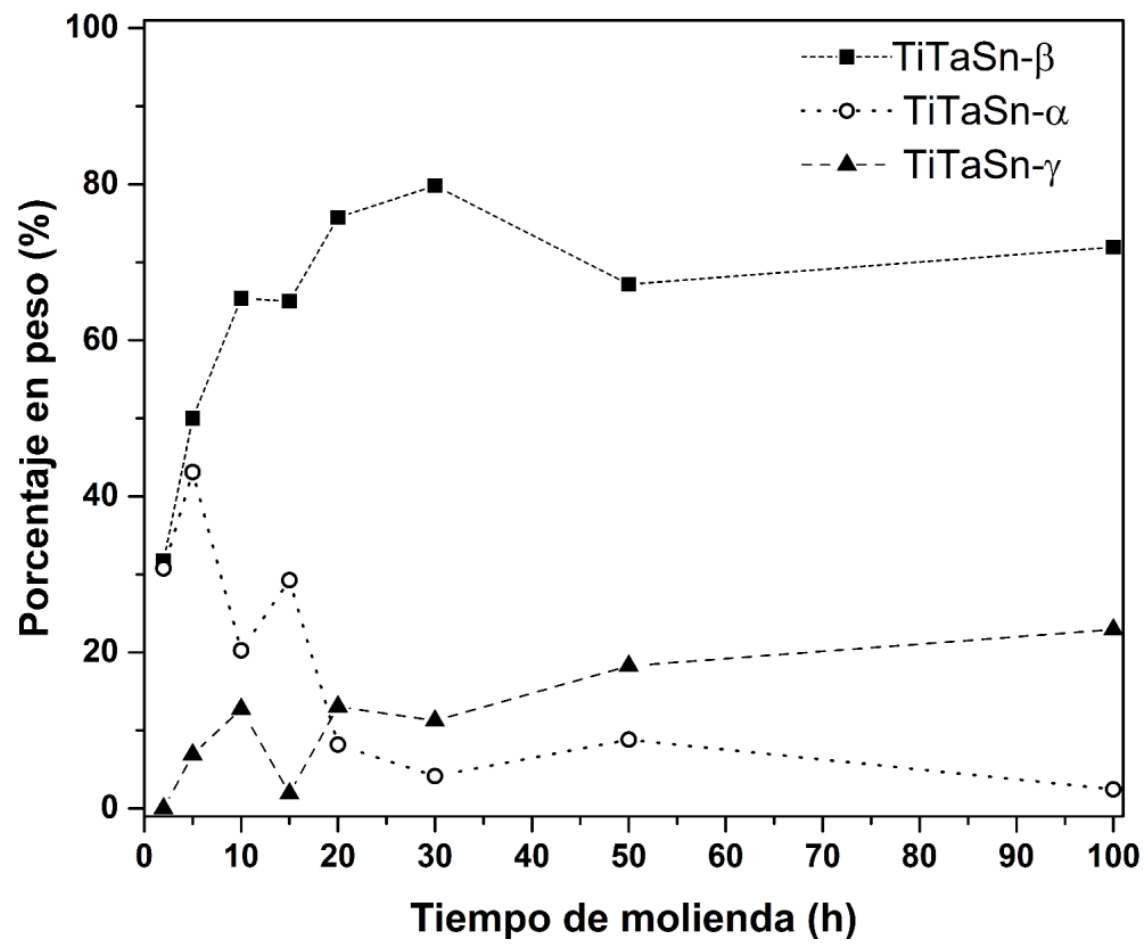

Figura 3: Evolución de las distintas fases de TiTaSn respecto de las horas de molienda.

Se aplicó el método Rietveld para determinar los valores de $\left(\left\langle\varepsilon^{2}\right\rangle^{1 / 2}\right)$ y tamaño de cristalita $(\langle\mathrm{D}\rangle)$, utilizando un modelo isotrópico para el tamaño y microdeformación. Esa información es relevante para analizar , la posibilidad de la recristalización mecánica, y saber qué fase se está formando [41-43]. En todos los refinamientos se obtuvieron valores de bondad de ajuste $\left(\chi^{2}\right)$ y $\mathrm{R}_{\mathrm{wp}}$ inferiores a 2 y $10 \%$, respectivamente, lo que muestra buena calidad del refinamiento $[44,45]$. En la Figura 4 se muestra un ejemplo del refinamiento de la muestra Ti-13Ta-3Sn (\%at.) a 30 horas de molienda, con valores de $\langle\mathrm{D}\rangle$ de $264,3 \mathrm{~nm}$ y $\left\langle\varepsilon^{2}\right\rangle^{1 / 2}$ de 0,0115 .

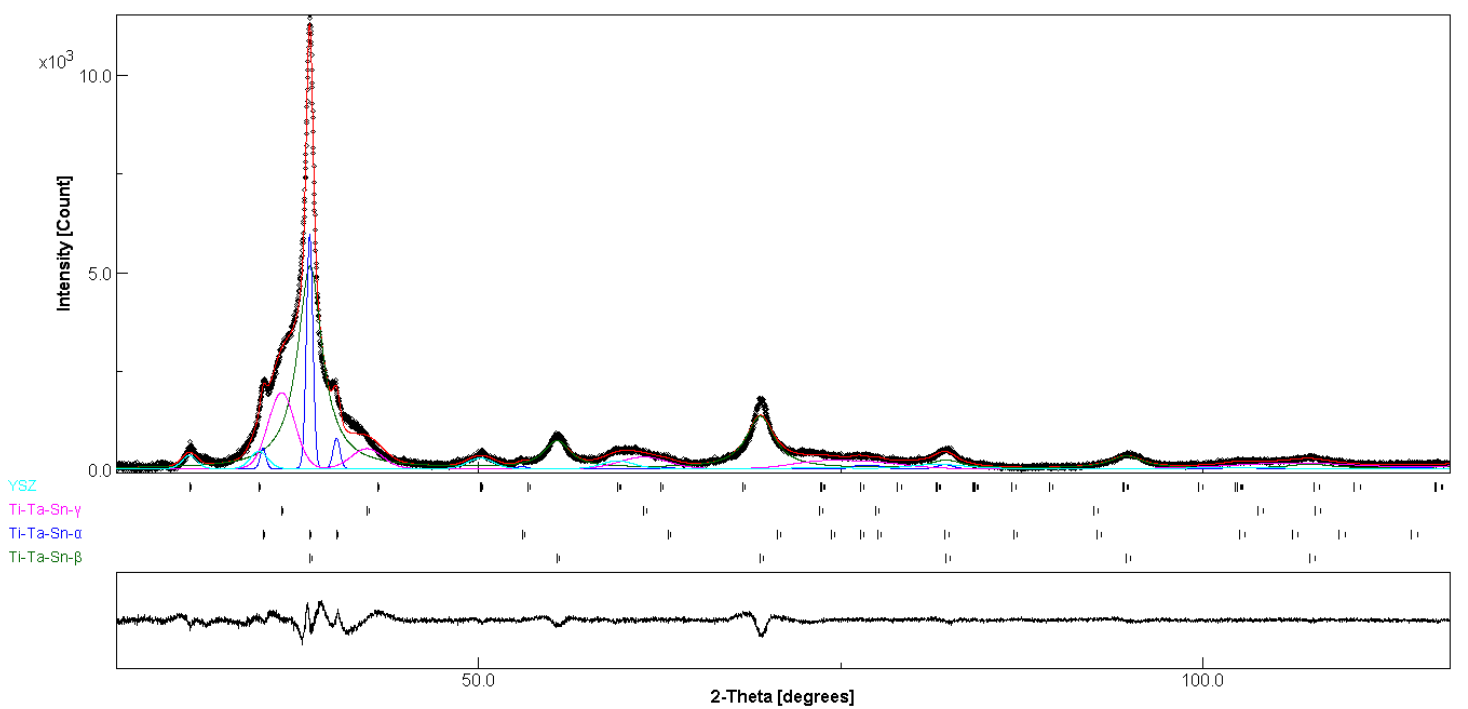

Figura 4: Refinamiento por el método Rietveld de la aleación ternaria Ti13\%Ta3\%Sn (\%at.) con 30h de molienda, usando los patrones de YSZ-st (P4 $\left.4_{2} / \mathrm{nmc}\right)$, TiTaSn- $\alpha\left(\mathrm{P6}_{3} / \mathrm{mmc}\right)$, TiTaSn- $\beta(\operatorname{Im} \overline{\mathbf{3}} \mathrm{m})$ y TiTaSn- $\gamma(\mathrm{Fm} \overline{\mathbf{3}} \mathrm{m})$.

La Figura 5 muestra la evolución de $\langle\mathrm{D}\rangle$ y el valor de $\left\langle\varepsilon^{2}\right\rangle^{1 / 2}$ de las fases presentes en función del tiempo de molienda. Como se observa en la Figura 3, a 10 horas de molienda, se identificó la trasformación de la fase TiTaSn- $\alpha$ a las fases TiTaSn- $\beta$ y TiTaSn- $\gamma$. Fenómeno, asociado al desplazamiento de los átomos causado por las altas microdeformaciones [53]. La fase TiTaSn- $\beta$ mostró el menor valor de tamaño de cristalita en un rango de 33 a $9 \mathrm{~nm}$ a $30 \mathrm{~h}$ de molienda. La fase TiTaSn- $\gamma$ mostro valores entre de 475 a $4 \mathrm{~nm}$ y 
finalmente la fase TiTaSn- $\alpha$ exhibió valores en un rango de 640 a $12 \mathrm{~nm}$. Entre 15 y 50 horas, las fases TiTaSn- $\alpha$ y TiTaSn- $\gamma$ presentaron recristalización mecánica, evidenciado en el incremento de tamaño de cristalita promedio. Después de las 50 horas de molienda, se tiene una disminución del tamaño de cristalita en las fases TiTaSn- $\alpha$ y TiTaSn- $\gamma$ y se observa que la fase TiTaSn- $\gamma$, llega al valor máximo de microdeformación. Mientras que para la fase TiTaSn- $\alpha$ a $30 \mathrm{~h}$ se observa un incremento en la microdeformación. Con respecto a la fase TiTaSn- $\beta$ se observa que a $2 \mathrm{~h}$ de molienda presenta el mayor valor $\left\langle\varepsilon^{2}\right\rangle^{1 / 2}(6,47 \mathrm{E}-4)$, luego disminuye hasta $9.33 \mathrm{E}-5$ para $30 \mathrm{~h}$ y aumenta levemente hasta $100 \mathrm{~h}$. A partir de $2 \mathrm{~h}$ se observa que el tamaño de cristalita disminuye desde $34 \mathrm{~nm} \mathrm{~nm}$ hasta un valor de $5 \mathrm{~nm} \mathrm{~nm}$ a $30 \mathrm{~h}$, para tiempos mayores de molienda el tamaño de cristalita alcanza un valor mínimo límite. Durante la molienda, la densidad de defectos cristalinos aumenta debido a los impactos de los medios de molienda, y el tamaño de cristalito alcanza un valor de saturación nanocristalino. Esto es debido a la dificultad de generar nuevas dislocaciones en tamaños de cristalitas nanocristalinas y debido a que algunas dislocaciones existentes son re-arregladas y eliminadas, por ello los valores de $<\varepsilon 2>1 / 2$ disminuyen. En el proceso de AM hay dos procesos que compiten, soldadura en frio y fractura de las partículas [54], por ello en fases con altos valores de $\left\langle\varepsilon^{2}\right\rangle^{1 / 2}$ el mecanismo de fractura es el predominante [55].
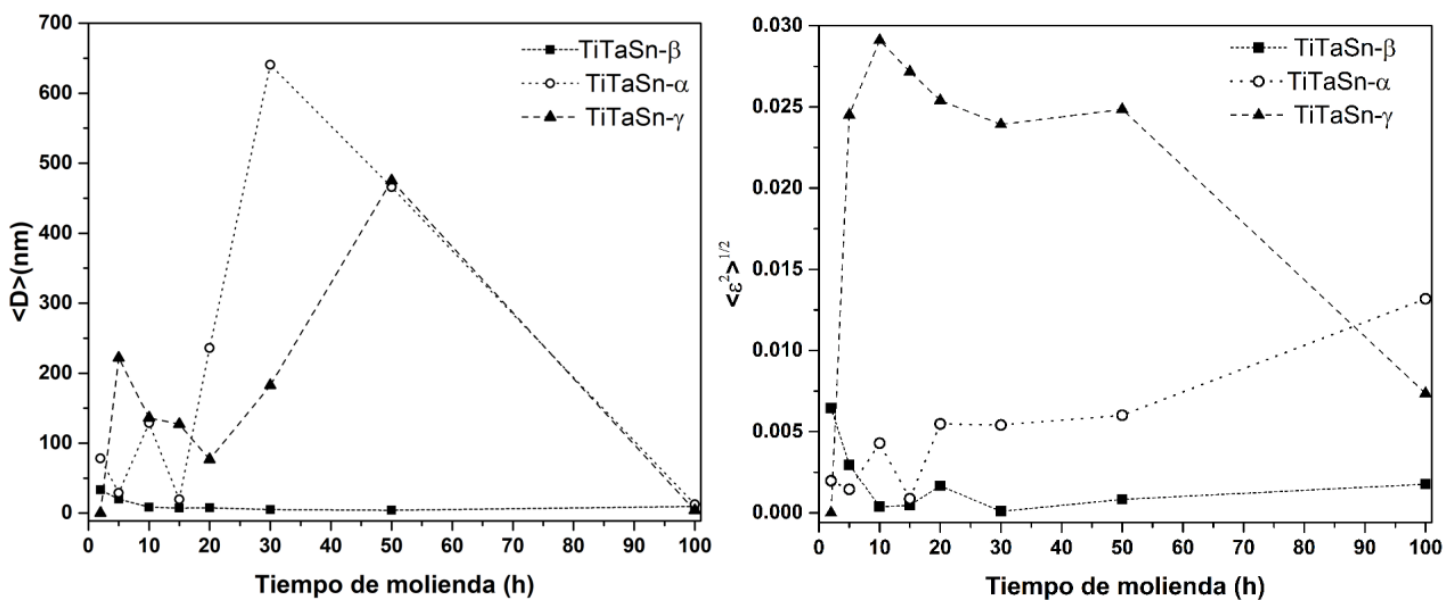

Figura 5: Evolución de tamaño de cristalita promedio (izquierda) y cuadrado de la raíz media de las microdeformaciones (derecha), de las fases TiTaSn- $\alpha$ (P6 $\left.{ }_{3} / \mathrm{mmc}\right)$, TiTaSn- $\beta$ (Im $\left.\overline{\mathbf{3}} \mathrm{m}\right)$ y fcc-TiTaSn- $\gamma(\mathrm{Fm} \overline{\mathbf{3}} \mathrm{m})$.

\section{DISCUSIÓN}

Los picos del Sn y Ta no están presentes por ello se asume que han entrado en solución sólida en fase alfa. A medida que aumenta el tiempo de molienda se identificó la trasformación de la fase TiTaSn- $\alpha$ a la fase TiTaSn- $\gamma$. E. Chicardi et al. [57] reportó una transformación similar en una aleación de TiNbMn obtenida por aleado mecánico. Ka Kohta Asano et al. [58] reportó una trasformación similar en una aleación binaria de $\mathrm{TiMg}$, adjudicando la formación de la fase Ti- $\gamma$ a las fallas de apilamiento de la estructura cristalina Ti-hcp. Los autores Partha et al. [59] e I. Mannaa et al. [60], reportaron transformación de Ti- $\alpha$ puro a Ti- $\gamma$, concluyendo que la deformación plástica es responsable de esta transformación. Por lo que, al incrementar las horas de molienda, se incrementaran las microdeformaciones de la fase TiTaSn- $\alpha$, lo cual promueva la transformación a la fase TiTaSn- $\gamma$, comportamiento apreciable después de las 20 horas de molienda (Figura 3 y Figura 5).

La desaparición de los picos del Sn y Ta entre 2 y 5 horas de molienda (Figura 2), promovió el incremento de la fase TiTaSn- $\beta$. Posterior a las 15 horas de molienda, se genera alta cantidad de fase TiTaSn- $\beta$, con una cantidad de $79,80 \%$ en peso a 30 horas de molienda (Figura 3). Los resultados reportados por Guo et al.[25], muestran que la aleación TiTa con adición de Sn promueve la estabilización de la fase TiTaSn- $\beta$, a partir de la adición de $3 \% \mathrm{Sn}$. Se alcanzó un estado estacionario para la formación de fase TiTaSn- $\beta$, posterior de las 30h de AM (Figura 5), lo cual está asociado a la saturación de la dureza y distribución constante del tamaño de partícula, de acuerdo con lo reportado por Benjamin y Volin [28]. 


\section{CONCLUSIONES}

A través del análisis de los patrones de difracción de rayos X y la aplicación del método Rietveld de la aleación TiTaSn. Se identificó que a las 2 horas de AM se inicia la transformación de la fase Ti- $\alpha$ a Ti- $\beta$, obteniendo un valor óptimo del 79,80\% en peso de fase Ti- $\beta$, a las 30 horas de molienda. Por otro lado, se observó que, a las 5 horas de AM, se presenta la formación de una nueva fase con estructura cubica centrada en las caras (TiTaSn- $\gamma$ ), cuantificándose una máximo del 22,93\% en peso, a las 100 horas de AM. Además de ello se determinaron los parámetros microestructurales de cada de una de estas fases, donde la fase Ti- $\beta$, mantuvo un tamaño promedio de cristalita entre un rango de 33 a $9 \mathrm{~nm}$, posterior a las 30 horas de molienda. Mientras que para la fase Ti- $\nu$, a un tiempo de 100 horas, logro alanzar un tamaño de $4 \mathrm{~nm}$.

\section{AGRADECIMIENTOS}

Los autores agradecen al apoyo de FONDECYT 1161444, FONDECYT 1190797, FONDEQUIP EQM 140095 y al Programa de Incentivos a la Iniciación Científica (PIIC) de la USM.

\section{BIBLIOGRAFÍA}

[1] Grupo Banco Mundial, "Esperanza de vida al nacer," 2018. [Online]. Available: https://datos.bancomundial.org/indicador/SP.DYN.LE00.IN. Accessed: 20-Oct-2018.

[2] International Osteoporosis Foundation, "Facts and Statistics," 2018. [Online]. Available: https://www.iofbonehealth.org/facts-statistics\#category-14. Accessed: 20-Oct-2018.

[3] MELTON, L. J., CHRISCHILlES E. A., COOPER C., et al., "Perspective how many women have osteoporosis?", Journal of Bone and Mineral Research, v. 7, n. 9, pp. 1005-1010, Dec. 2009.

[4] MELTON, L. J., ATKINSON, E. J, O’CONNOR, M. K., et al., "Bone Density and Fracture Risk in Men,' Journal of Bone and Mineral Research, v. 13, no. 12, pp. 1915-1923, Dec. 1998.

[5] KANIS, J. A., et al., "Long-term risk of osteoporotic fracture in Malmö”, Osteoporosis international : a journal established as result of cooperation between the European Foundation for Osteoporosis and the National Osteoporosis Foundation of the USA, v. 11, n. 8, pp. 669-74, 2000.

[6] LONG, M., RACK, H. J., "Titanium alloys in total joint replacement - A materials science perspective," Biomaterials, v. 19, n. 18, pp. 1621-1639, Sep. 1998.

[7] STEINEMANN, K. G. S.G., WINTER, IN G.D., LERAY, J.L., "Corrosion of surgical implants -in vivo and in vitro tests", Evaluation of Biomaterials, v. 1, pp. 1-34, 1980.

[8] STEINEMANN S. G., "Corrosion of titanium and titanium alloys for surgical implants", Titanium-Science and Technology, v. 2, pp. 1373-1379, 1984.

[9] JOHNSTONE, T., “Aluminum and Alzheimer's disease”, CMAJ : Canadian Medical Association journal = journal de l'Association medicale canadienne, v. 146, n. 4, pp. 431-2, Feb. 1992.

[10] CRAPPER MCLACHLAN, D. R., “Aluminum and alzheimer's disease”, Neurobiology of Aging, v. 7, no. 6, pp. 525-532, Nov. 1986.

[11] NIINOMI, M., "Recent titanium R\&amp;D for biomedical applications in japan”, Jom, v. 51, n. 6, pp. 32-34, 1999.

[12] TU M, E., WU, Y. H., "Multiple allergies to metal alloys", Dermatologica Sinica, v. 29, n. 2, pp. 41-43, Jun. 2011.

[13] GIESEN, E. B. W., DING, M., DALSTRA, M., et al., "Mechanical properties of cancellous bone in the human mandibular condyle are anisotropic", Journal of Biomechanics, v. 34, n. 6, pp. 799-803, Jun. 2001.

[14] MURPHY W., BLACK J., HASTINGS G., Eds., Handbook of Biomaterial Properties, New York, NY, Springer New York, 2016.

[15] HERMAN, I. P., Physics of the Human Body, 2016.

[16] FROES, F. H., Titanium : physical metallurgy, processing, and applications.

[17] DONACHIE M. J., DONACHIE S. J., A technical guide, ASM International, 2002.

[18] MOHAMMED M. T., KHAN Z. A., SIDDIQUEE A. N., "Beta-Titanium-Alloys-The-Lowest-ElasticModulus-for-Biomedical-Applications-A-Review", International Journal of Chemical, v. 8, n. 8, pp. 822$827,2014$.

[19] GEETHA M., SINGH A. K., ASOKAMANI R., et al., "Ti based biomaterials, the ultimate choice for orthopaedic implants - A review", Progress in Materials Science, v. 54, n. 3, Pergamon, pp. 397-425, 01May-2009. 
[20] NIINOMI, M., et al., "Corrosion wear fracture of new $\beta$ type biomedical titanium alloys", Materials Science and Engineering A, v. 263, n. 2, pp. 193-199, May 1999.

[21] KHAN, M. A., WILLIAMS, R. L., WILLIAMS, D. F., "In-vitro corrosion and wear of titanium alloys in the biological environment”, v. 17, n. 22, pp. 2117-2126, 1996.

[22] KAWAHARA, H., "Cytotoxicity of Implantable Metals and Alloys", Bulletin of the Japan Institute of Metals, v. 31, n. 12, pp. 1033-1039, Dec. 1992.

[23] PAPAKYRIACOU M., MAYER H., PYPEN C., et al., "Effects of surface treatments on high cycle corrosion fatigue of metallic implant materials", International Journal of Fatigue, v. 22, n. 10, pp. 873-886, Nov. 2000.

[24] MIKI M., MORITA M., "Evaluation of the biocompatibility of a Ti-Ta-Sn alloy using cell cultures.," Materials Transactions, v. 56, n. 7, pp. 1087-1091, Jul. 2015.

[25] GUO B., TONG Y. X., CHEN F., et al., "Effect of Sn addition on the corrosion behavior of Ti-Ta alloy," Materials and Corrosion, v. 63, n. 3, pp. 259-263, Mar. 2012

[26] LU L., LAI M. O., ZHANG S., "Diffusion in mechanical alloying”, Journal of Materials Processing Technology, v. 67, n. 1-3, pp. 100-104, May 1997.

[27] SCHAFFER, G. B., MCCORMICK, P. G., "Displacement reactions during mechanical alloying", Metallurgical Transactions A, v. 21, n. 10, pp. 2789-2794, Oct. 1990.

[28] BENJAMIN, J. S., VOLIN, T. E., “The mechanism of mechanical alloying”, Metallurgical Transactions, v. 5, n. 8, pp. 1929-1934, Aug. 1974.

[29] LU L., LAI M. O., "Formation of new materials in the solid state by mechanical alloying", Materials \& Design, v. 16, n. 1, pp. 33-39, Jan. 1995.

[30] LIU L., PADELLA F., GUO W., et al., "Solid state reactions induced by mechanical alloying in metalsilicon (metal = Mo, Nb) systems", Acta Metallurgica Et Materialia, v. 43, n. 10, pp. 3755-3761, Oct. 1995.

[31] KOCH, C.C., "Intermetallic matrix composites prepared by mechanical alloying-a review", Materials Science and Engineering: A, v. 244, n. 1, pp. 39-48, Mar. 1998.

[32] KOCH C. C., WHITTENBERGER J. D., "Mechanical milling/alloying of intermetallics", Intermetallics, v. 4, n. 5. Elsevier, pp. 339-355, 01-Jan-1996.

[33] FECHT H. J., HAN G., FU Z., et al., "Metastable phase formation in the Zr-Al binary system induced by mechanical alloying”, Journal of Applied Physics, v. 67, n. 4, pp. 1744-1748, 1990.

[34] OEHRING, M., YAN, Z. H., KLASSEN, T., et al., "Competition between stable and metastable phases during mechanical alloying and ball milling”, physica status solidi (a), v. 131, n. 2, pp. 671-689, Jun. 1992.

[35] MA, E., ATZMON, M., "Phase transformations induced by mechanical alloying in binary systems", Materials Chemistry \& Physics, v. 39, n. 4. Elsevier, pp. 249-267, 31-Jan-1995.

[36] SCHWARZ, R. B., KOCH, C. C., "Formation of amorphous alloys by the mechanical alloying of crystalline powders of pure metals and powders of intermetallics", Applied Physics Letters, v. 49, n. 3, pp. 146-148, 1986.

[37] ECKERT, J., SCHULTZ, L., URBAN, K., “Amorphization reaction during mechanical alloying: influence of the milling conditions”, Journal of Materials Science, v. 26, n. 2, pp. 441-446, 1991.

[38] HELLSTERN E., SCHULTZ L., "Glass formation in mechanically alloyed transition metal - titanium alloys”, Materials Science and Engineering, v. 93, pp. 213-216, Sep. 1987.

[39] ROJAS, P. A., MARTÍNEZ, C., AGUILAR, C., et al., "Caracterización de los cambios de fases en la fabricación de aleaciones base cobre, cristalinas y no cristalinas, por aleado mecánico", Ingenieria e Investigacion, v. 36, n. 3, pp. 102-109, Dec. 2016.

[40] PELEGRINI, L., BITTENCOURT, S. D., PAUlETTI, P., et al., "Influence of Milling Time During the Mechanical Alloying Process on the Properties of Fe-3Si-0. 75P Alloy 2 . Experimental Procedures", Materials Research, v. 18, n. 5, pp. 1070-1076, Oct. 2015.

[41] LUTTEROTTI, L., MATTHIES, S., WENK, H.-R., "MAUD: a friendly Java program for Material Analysis Using Diffraction", IUCr: Newsletter of the CPD, v. 21, n. 14, p. 15, 1999.

[42] LUTTEROTTI, L., SCARDI, P., "Simultaneous structure and size-strain refinement by the Rietveld method", Journal of Applied Crystallography, v. 23, n. 4, pp. 246-252, Aug. 1990.

[43] SCARDI, P., LUTTEROTTI, L., MAISTRELLI, P., "Experimental determination of the instrumental broadening in the Bragg-Brentano geometry", Powder Diffraction, v. 9, n. 3, pp. 180-186, Sep. 1994.

[44] MEI, W., SUN, J., WEN, Y., "Martensitic transformation from $\beta$ to $\alpha^{\prime}$ and $\alpha$ " phases in Ti-V 
alloys: A first-principles study", Journal of Materials Research, v. 32, n. 16, pp. 3183-3190, Aug. 2017.

[45] KASSAN-OGLY F. A., ARKHIPOV V. E., SHESTAKOV A. E., "Phase transitions in crystals with a BCC structure," The Physics of Metals and Metallography, v. 109, n. 6, pp. 568-584, Jun. 2010.

[46] WANG Q., LIU Z., WANG B., HASSAN MOHSAN A. U., "Stress-induced orientation relationship variation for phase transformation of $\alpha$-Ti to $\beta$-Ti during high speed machining Ti-6Al-4V," Materials Science and Engineering: A, v. 690, pp. 32-36, Apr. 2017.

[47] HONG D. H., LEE T. W., LIM S. H., et al., "Stress-induced hexagonal close-packed to face-centered cubic phase transformation in commercial-purity titanium under cryogenic plane-strain compression", Scripta Materialia, v. 69, n. 5, pp. 405-408, Sep. 2013.

[48] AL-AQEELI N., SURYANARAYANA C., HUSSEIN M. A., "Formation of an amorphous phase and its crystallization in the immiscible $\mathrm{Nb}-\mathrm{Zr}$ system by mechanical alloying", Journal of Applied Physics, v. 114, n. 15, p. 153512, Oct. 2013.

[49] PATIL U., HONG S. J., SURYANARAYANA C., "An unusual phase transformation during mechanical alloying of an Fe-based bulk metallic glass composition", Journal of Alloys and Compounds, v. 389, n. 1-2, pp. 121-126, Mar. 2005.

[50] SHARMA, S., SURYANARAYANA, C., "Mechanical crystallization of Fe-based amorphous alloys," Journal of Applied Physics, v. 102, n. 8, p. 083544, Oct. 2007.

[51] MCCUSKER L. B., VON DREELE R. B., COX D. E., et al., "Rietveld refinement guidelines," International Union of Crystallography Journal of Applied Crystallography", J. Appl. Cryst, v. 32, pp. 36-50, 1999.

[52] WILL, G., "Powder Diffraction - The Rietveld Method and the Two Stage Method to Determine and Refine Crystal Structures from Powder Diffraction Data", Springer-Verlag Berlin Heidelberg. Springer, 2006.

[53] KHINA, B. B., SOLPAN, I., LOVSHENKO, G. F., "Modelling accelerated solid-state diffusion under the action of intensive plastic deformation", Journal of Materials Science, , v. 39, n. 16-17, pp. 5135-5138, 2004.

[54] SURYANARAYANA, C., "Mechanical alloying and milling", Progress in Materials Science, v. 46, n. 1-2. Pergamon, pp. 1-184, 01-Jan-2001.

[55] AGUILAR C., GUZMÁN D., CASTRO F., et al., "Fabrication of nanocrystalline alloys Cu-Cr-Mo super satured solid solution by mechanical alloying," Materials Chemistry and Physics, vol. 146, no. 3, pp. 493-502, Aug. 2014.

[56] FERNÁNDEZ-COLUMBIÉ, T., RODRÍGUEZ-GONZÁLEZ, I., QUINTANA-PUCHOL, R., et al., Ingeniería Mecánica., vol. 15, no. 1. Facultad de Ingeniería Mecánica. Instituto Superior Politécnico "José Antonio Echeverría," 2012.

[57] CHICARDI E., GARCÍA-GARRIDO C., SAYAGUÉS M. J., et al., "Development of a novel fcc structure for an amorphous-nanocrystalline Ti-33Nb-4Mn (at.\%) ternary alloy", Materials Characterization, v. 135, pp. 46-56, Jan. 2018.

[58] ASANO, K., ENOKI, H., AKIBA, E., "Synthesis of HCP, FCC and BCC structure alloys in the Mg-Ti binary system by means of ball milling", Journal of Alloys and Compounds, v. 480, n. 2, pp. 558-563, Jul. 2009.

[59] CHATTERJEE P., SEN GUPTA, S. P., "An X-ray diffraction study of strain localization and anisotropic dislocation contrast in nanocrystalline titanium", Philosophical Magazine A, v. 81, n. 1, pp. 49-60, Jan. 2001.

[60] MANNA I., CHATTOPADHYAY P. P., NANDI P., et al., "Formation of face-centered-cubic titanium by mechanical attrition", Journal of Applied Physics, v. 93, n. 3, pp. 1520-1524, Feb. 2003.

\section{ORCID}

Edgar Ivan Pio Lopez

Pablo Ignacio Martin Saint-Laurence

Claudio Eduardo Aguilar Ramirez

Ariosto Medina Flores

Fernando de las Cuevas Jiménez

Ismeli Alfonso López

Luis Bejar Gomez https://orcid.org/0000-0002-3962-4326

https://orcid.org/0000-0001-7158-8620

https://orcid.org/0000-0002-9013-5835

https://orcid.org/0000-0002-9709-8790

https://orcid.org/0000-0002-2344-7353

https://orcid.org/0000-0001-7311-8614

https://orcid.org/0000-0002-5954-6839 